\title{
Analytical Approximation for Distorted Expectations
}

\author{
Xianming Sun ${ }^{* a, b}$, Siqing Gan ${ }^{\mathrm{a}}$, Michèle Vanmaele ${ }^{\mathrm{b}}$ \\ ${ }^{a}$ School of Mathematics and Statistics, Central South University, Changsha, Hunan, 410004, China \\ ${ }^{b}$ Department of Applied Mathematics, Computer Science and Statistics, Ghent University, Krijgslaan 281 S9, 9000 Gent, \\ Belgium
}

\begin{abstract}
Given the density function or the characteristic function of a random variable, we propose an analytical approximation for its distorted expectation by using the fast Fourier transform algorithm. This approach can be used in various applications involving distorted expectations.
\end{abstract}

Keywords: Distorted expectations, Fourier-cosine series expansion, Coherent risk measure

\section{Introduction}

This paper proposes an accurate and efficient numerical method to calculate the distorted expectation of the following form,

$$
\mathbb{E}_{\Psi}[X]=\int_{-\infty}^{+\infty} x d \Psi\left(F_{X}(x)\right)
$$

where $X$ is the risk factor of interest with distribution function $F_{X}$ and $\Psi$ is a distortion function.

Distorted expectations arise in different branches in the area of insurance and finance [see e.g. 22, 3, 7, 16, $12,8,15,20,1,21,23$, to name a few]. If any, quite few models for the risk factor $X$ admit an analytical formula for the distorted expectation with respect to a distortion function in the general setting. The Monte Carlo $(\mathrm{MC})$ method is a standard way to calculate the distorted expectations. If $\left\{x_{1}, \ldots, x_{N}\right\}$ is a set of MC realizations of the risk factor $X$, or its sample from market data, the value of the distorted expectation $\mathbb{E}_{\Psi}[X]$ can be estimated by

$$
\mathbb{E}_{\Psi}[X] \doteq \sum_{n=1}^{N} x_{(n)}\left(\Psi\left(\frac{n}{N}\right)-\Psi\left(\frac{n-1}{N}\right)\right)
$$

where $x_{(1)}, \ldots, x_{(N)}$ are the values of $x_{1}, \ldots, x_{N}$ in the increasing order [7]. To achieve high accuracy, a large ordered sample of the risk factor is required. This procedure can be time-consuming under complex models for the risk factor, such as the Heston model [13]. On the other hand, some efficient numerical methods for the standard expectations, such as the COS method [9] and the Carr-Madan method [5], can hardly be used to calculate the distorted expectations, because there is no analytical formula for the distorted characteristic function in most of the realistic cases.

To overcome these difficulties, we propose an analytical approximation method, as an alternative to the MC method, for a class of distorted expectations. In applications, the parametric model for the risk factor $X$ usually admits the density function $f_{X}$ or the characteristic function $\phi_{X}$ in an analytical form. Then, the distribution function $F_{X}$ can be numerically calculated or recovered from its characteristic function. In this paper, we denote the distorted density function $\tilde{f}_{X}$ by $\left(\Psi^{\prime} \circ F_{X}\right) \cdot f_{X}$ with $\tilde{f}_{X}(x)=\Psi^{\prime}\left(F_{X}(x)\right) f_{X}(x), x \in \mathbb{R}$. The fast Fourier transform (FFT) algorithm is used to set up an approximation for $\tilde{f}_{X}$ with a truncated sum of its Fourier-cosine series expansion on a finite interval. The resulting truncation approximation immediately provides an analytical approximation for the distorted expectations.

The remainder of this paper is organized as follows. Preliminaries on the distorted expectation and its relation with coherent risk measures are provided in Section 2. Section 3 introduces the analytical approximation of the distorted expectations. Numerical examples to calculate the distorted expectation of asset price in the Heston model are carried out in Section 4, and Section 5 concludes.

\section{Preliminaries}

Let $\left(\Omega, \mathcal{F},\left(\mathcal{F}_{t}\right)_{t \in[0, T]}, P\right)$ be a filtered probability space. Denote by $L^{2}(\Omega, \mathcal{F}, P)$ the collection of squareintegrable random variables representing the profits and losses of a financial position. The risk of a random

*Corresponding author. Email: Xianming.Sun@hotmail.com. Tel. +32 092644773. 
variable $X \in L^{2}(\Omega, \mathcal{F}, P)$ can be quantified with a risk measure $\rho$, a functional defined on $L^{2}(\Omega, \mathcal{F}, P)$. Probability distortion provides a large class of risk measures.

\subsection{Distorted Expections}

Definition 2.1. Let $\Psi:[0,1] \rightarrow[0,1]$ be a continuous increasing function such that $\Psi(0)=0$ and $\Psi(1)=1$. The set function $c_{\Psi}$ defined by

$$
c_{\Psi}(A)=\Psi(P(A)), \quad A \in \mathcal{F},
$$

is called the distortion of the probability measure $P$ with respect to the distortion function $\Psi$.

With a probability distortion function $\Psi$ is associated another probability distortion function $\hat{\Psi}$ given by

$$
\hat{\Psi}(x)=1-\Psi(1-x), \quad x \in[0,1] .
$$

Given a probability distortion $\Psi$, we can define the Choquet integral $\mathbb{E}_{\Psi}[X]$ of $X \in L^{2}(\Omega, \mathcal{F}, P)$ by

$$
\mathbb{E}_{\Psi}[X]=\int_{0}^{\infty}\left(1-c_{\Psi}(\{X \leq x\})\right) d x-\int_{-\infty}^{0} c_{\Psi}(\{X \leq x\}) d x .
$$

We assume that $\int_{0}^{1} \Psi(y) \frac{d y}{2 y \sqrt{y}}<\infty$ and the probability space $(\Omega, \mathcal{F}, P)$ is atomless throughout this paper. Then, according to [17], $\mathbb{E}_{\Psi}[X]$ is finite for any $X \in L^{2}(\Omega, \mathcal{F}, P)$ when $\Psi$ is concave and continuous on $[0,1]$.

Let $F_{X}$ be the distribution of $X$. We can rewrite $\mathbb{E}_{\Psi}[X]$ as

$$
\mathbb{E}_{\Psi}[X]=\int_{-\infty}^{+\infty} x d \Psi\left(F_{X}(x)\right) .
$$

$\mathbb{E}_{\Psi}$ refers to a distorted expectation associated with a distortion function $\Psi$. If $\Psi(x)=x, \mathbb{E}_{\Psi}$ is the standard expectation $E$.

For any $X \in L^{2}(\Omega, \mathcal{F}, P)$, the distorted expectation $\mathbb{E}_{\Psi}[X]$ associated with a concave and continuous distortion function $\Psi$ admits a robust representation

$$
\mathbb{E}_{\Psi}[X]=\inf _{Q \in \mathcal{Q}_{\Psi}} E^{Q}[X]
$$

where

$$
\mathcal{Q}_{\Psi}=\left\{Q \in \mathcal{M}_{P}: \hat{\Psi}(P(A)) \leq Q(A) \leq \Psi(P(A)) \text { for all } A \in \mathcal{F}\right\},
$$

with $\mathcal{M}_{P}$ being the collection of all probability measures absolutely continuous with respect to $P$ [17].

\subsection{Coherent risk measures}

Definition 2.2. A coherent risk measure on $L^{2}(\Omega, \mathcal{F}, P)$ is a map $\rho: L^{2}(\Omega, \mathcal{F}, P) \rightarrow \mathbb{R}$ satisfying the following properties:

1. (subadditivity) for $X, Y \in L^{2}(\Omega, \mathcal{F}, P), \rho(X+Y) \leq \rho(X)+\rho(Y)$;

2. (monotonicity) If $X \leq Y$ a.s., then $\rho(X) \leq \rho(Y)$;

3. (positive homogeneity) $\rho(\lambda X)=\lambda \rho(X)$ for $\lambda \in \mathbb{R}_{+}$;

4. (translation invariance) $\rho(X+m)=\rho(X)-m$ for $m \in \mathbb{R}$;

A coherent risk measure $\rho$ defined on the space $L^{\infty}(\Omega, \mathcal{F}, P)$ of all bounded random variables $(a . s$.$) , has a$ robust representation [2]

$$
\rho(X)=\sup _{Q \in \mathcal{Q}} E^{Q}[-X]=-\inf _{Q \in \mathcal{Q}} E^{Q}[X], \quad \text { for } X \in L^{\infty}(\Omega, \mathcal{F}, P),
$$

where $\mathcal{Q}$ is a subset of $\mathcal{M}_{1, f}(\Omega, \mathcal{F})$, the set of all finitely additive normalized set functions $Q: \mathcal{F} \rightarrow[0,1] . \mathcal{Q}$ can be chosen as a convex set for which the supremum or infimum is attained [Proposition 4.15 of 10]. This robust representation can be generalized to the risk measures defined on the space $L^{0}(\Omega, \mathcal{F}, P)$ of all random variables [6]. Recalling the robust representation of the distorted expectation $(2.3)$ defined on $L^{2}(\Omega, \mathcal{F}, P)$, we can identify the relation between risk measures and distorted expectations as follows:

$$
\rho(X):=-\mathbb{E}_{\Psi}[X], \quad X \in L^{2}(\Omega, \mathcal{F}, P),
$$

is a coherent risk measure if $\Psi$ is concave and continuous on $[0,1]$. The Average Value at Risk $(A V @ R)$ is an example of the distortion risk measures [10]. 
Example 2.1 (Average Value at Risk). Let $\mathcal{Q}_{\lambda}$ be the class of all probability measures $Q \ll P$ whose density $d Q / d P$ is bounded by $1 / \lambda$ for some fixed parameter $\lambda \in(0,1)$. The coherent risk measure

$$
A V @ R_{\lambda}(X):=\sup _{Q \in \mathcal{Q}_{\lambda}} E^{Q}[-X]
$$

is so-called the Average Value at Risk. Actually,

$$
A V @ R_{\lambda}(X)=-\frac{1}{\lambda} \int_{0}^{\lambda} q_{X}(t) d t
$$

where $q_{X}$ is the quantile function of $X$. Induced by a distorted expectation, $A V @ R_{\lambda}(X)=-\mathbb{E}_{\Psi^{\lambda}}[X]$ where

$$
\Psi^{\lambda}(x)=\left(\frac{x}{\lambda}\right) \wedge 1, \quad x \in[0,1] .
$$

To complete this section, we outline some other risk measures induced by their corresponding distortion functions [7].

Table 1: Distortion risk measures and the corresponding distortion functions

\begin{tabular}{|l|c|l|}
\hline & $\Psi^{\lambda}(x)(\lambda>0)$ & if $\lambda \in \mathbb{N}^{+}, \mathbb{E}_{\Psi^{\lambda}}[X]=E[Y]$, where $Y$ is a random variable. \\
\hline MAXMINV@R & $\left(1-(1-x)^{\lambda+1}\right)^{\frac{1}{1+\lambda}}$ & $\begin{array}{l}\max \left\{Y_{1}, \ldots, Y_{\lambda+1}\right\} \stackrel{\text { law }}{=} \min \left\{X_{1}, \ldots, X_{\lambda+1}\right\}, \\
\text { where }\left\{X_{1}, \ldots, X_{\lambda+1}\right\} \text { are independent draws of } X \\
\text { and }\left\{Y_{1}, \ldots, Y_{\lambda+1}\right\} \text { are independent draws of } Y .\end{array}$ \\
\hline MINMAXV@R & $1-\left(1-x^{\frac{1}{\lambda+1}}\right)^{\lambda+1}$ & $\left\{\begin{array}{l}Y \stackrel{\operatorname{law}}{=} \min \left\{Z_{1}, \ldots, Z_{\lambda+1}\right\} \\
\max \left\{Z_{1}, \ldots, Z_{\lambda+1}\right\} \stackrel{\text { law }}{=} X \\
\text { where }\left\{Z_{1}, \ldots, Z_{\lambda+1}\right\} \text { are independent draws of } Z .\end{array}\right.$ \\
\hline
\end{tabular}

\section{Analytical approximation for distorted expectations}

Since the density function of the risk factor $X$ can be estimated with statistical methods or recovered from its characteristic function, without loss of generality, we assume the density function $f_{X}$ is given in its analytical form. Then, its distribution function can be calculated either by numerical integration methods or recovered from its characteristic function $[18,19]$. Hence, we actually assume that both the density function and the distribution function are known when formulating the proposed approach.

Given the distribution function $F_{X}$ and the distortion function $\Psi$, we can, theoretically, derive the distorted distribution function $\tilde{F}_{X}$ and the distorted density function $\tilde{f}_{X}$ as

$$
\left\{\begin{aligned}
\tilde{F}_{X}(x) & =\Psi\left(F_{X}(x)\right), & & x \in \mathbb{R}, \\
\tilde{f}_{X}(x) & =\Psi^{\prime}\left(F_{X}(x)\right) f_{X}(x), & & x \in \mathbb{R} .
\end{aligned}\right.
$$

However, (3.1) can hardly lead to an analytical formula for the distorted expectation (2.2). Inspired by the COS method [9], we first truncate the integration interval of the distorted expectation into a finite interval $[a, b]$ such that

$$
\int_{(-\infty, a) \cup(b,+\infty)} x \tilde{f}_{X}(x) d x<\varepsilon, \quad \text { for a given tolerance error } \varepsilon>0 .
$$
by

Define $\hat{f}_{X}=\tilde{f}_{X} \mathbf{1}_{[a, b]}$, where $\mathbf{1}_{[a, b]}$ is an indicator function. The Fourier-cosine series expansion of $\hat{f}_{X}$ is given

$$
\hat{f}_{X}(x)=\sum_{k=0}^{\infty} \hat{F}_{k} \cdot \cos \left(k \pi \frac{x-a}{b-a}\right) .
$$

We propose to approximate the $\hat{f}_{X}$ by the truncated sum $\bar{f}_{X}$ of a Fourier-cosine series expansion, i.e.,

$$
\hat{f}_{X}(x) \approx \bar{f}_{X}(x)=\sum_{k=0}^{K} \hat{F}_{k} \cdot \cos \left(k \pi \frac{x-a}{b-a}\right) .
$$

The following two propositions characterize the approximation error and convergence order of the truncation approximation (3.3). Their proofs can be found in Chapter 2 of [4]. 
Proposition 3.1. The error in approximating $\hat{f}_{X}(x)(3.2)$ by the partial sum $\bar{f}_{X}(x)(3.3)$ is bounded by the sum of absolute values of all the neglected coefficients. That is,

$$
\left|\hat{f}_{X}(x)-\bar{f}_{X}(x)\right| \leq \sum_{k=K+1}^{\infty}\left|\hat{F}_{k}\right|, \quad x \in[a, b] .
$$

Proposition 3.2. If $\hat{f}_{X}$ is infinitely differentiable with nonzero derivatives on $[a, b]$, its Fourier-cosine series expansion has geometric convergence, i.e.,

$$
\hat{F}_{k} \sim O\left(k^{-n} \exp (-\gamma k)\right)
$$

where $\gamma$ is determined by the location in the complex plane of the singularities nearest to the expansion interval, and $n$ is determined by the type and strength of the singularity. Otherwise, the convergence of Fourier cosine series is algebraic, i.e.,

$$
\hat{F}_{k} \sim O\left(1 / k^{n}\right),
$$

where $n$ is at least as large as the highest order of derivative that exists or is nonzero.

Proposition 3.2 implies that the right (left) truncation bound $b(a)$ should not be too large (small). Otherwise, $\hat{f}_{X}$ may have zero derivatives, and the accuracy of the truncation approximation may be decreased. We suggest to check the tails of the original density function before selecting the truncation interval $[a, b]$.

The key procedure to set up the truncation approximation (3.3) is to calculate the coefficients $\hat{F}_{k}$, which can be calculated with the FFT algorithm as follows (see e.g. [11] for a theoretical derivation).

Algorithm 3.1 (The FFT algorithm for $\hat{F}_{k}$ ).

1. Calculate the value $\mathbf{Y}=\left(Y_{0}, \ldots, Y_{K}\right)$ of the distorted density function on the interpolation points $\mathbf{y}=$ $\left(y_{0}, \ldots, y_{K}\right)$, i.e.,

$$
\mathbf{Y}=\Psi^{\prime}\left(F_{X}(\mathbf{y})\right) f_{X}(\mathbf{y}), \quad \text { with } y_{k}=a+(b-a) k / K, \quad k \in\{0,1, \ldots, K\} .
$$

2. Extend $\mathbf{Y}$ to $\tilde{\mathbf{Y}}=\left(Y_{0}, Y_{1}, \ldots, Y_{k-2}, Y_{K-1}, Y_{K}, Y_{K-1}, Y_{K-2}, \ldots, Y_{2}, Y_{1}\right)$.

3. Apply the FFT algorithm to $\tilde{\mathbf{Y}}$, and denote the Fourier transform of $\tilde{\mathbf{Y}}$ by $\overline{\mathbf{Y}}$, a $2 K$-dimensional vector.

4. Then the coefficients are given by $\hat{F}_{k}=\bar{Y}_{k}$ if $k=1, \ldots, K-1 ; \hat{F}_{0}=0.5 \bar{Y}_{0} ; \hat{F}_{K}=0.5 \bar{Y}_{K}$.

After (3.3) is set up, the distorted expectation $\mathbb{E}_{\Psi}[X]$ can be approximated by

$$
\mathbb{E}_{\Psi}[X]=\int_{-\infty}^{\infty} x \tilde{f}_{X}(x) d x \approx \int_{a}^{b} x \bar{f}_{X}(x) d x=\int_{a}^{b} x \sum_{k=0}^{K} \hat{F}_{k} \cdot \cos \left(k \pi \frac{x-a}{b-a}\right) d x=\sum_{k=0}^{K} \hat{F}_{k} V_{k},
$$

with

$$
V_{k}=\int_{a}^{b} x \cos \left(k \pi \frac{x-a}{b-a}\right) d x=\frac{(b-a)^{2}}{(k \pi)^{2}}(\cos (k \pi)-1), \quad 1 \leq k \leq K ; \quad V_{0}=0.5\left(b^{2}-a^{2}\right) .
$$

We call the truncated approximation (3.4) as the T-COS approximation for the distorted expectation $\mathbb{E}_{\Psi}[X]$.

The truncation approximation (3.3) can be easily extended to calculate the distorted expectation of a combination of risk factors $\left(X_{1}, \ldots, X_{n}\right)$ with joint characteristic function $\phi(\boldsymbol{\omega})$,

$$
\phi(\boldsymbol{\omega})=E\left[e^{i\left(\omega_{1} X_{1}+\cdots+\omega_{n} X_{n}\right)}\right], \quad \text { where } \boldsymbol{\omega}=\left(\omega_{1}, \ldots, \omega_{n}\right) .
$$

In fact, the characteristic function of a portfolio $X=z_{1} X_{1}+\cdots+z_{n} X_{n}$ is

$$
\phi_{X}(z)=E\left[e^{i z\left(z_{1} X_{1}+\cdots+z_{n} X_{n}\right)}\right]=\phi(\boldsymbol{\omega}), \quad \text { with } \boldsymbol{\omega}=\left(z z_{1}, \ldots, z z_{n}\right) .
$$

Hence, the density function and the distribution function can be recovered from the characteristic function $\phi_{X}$ with the COS method [18, 19].

\section{Numerical examples}

The Heston model [13] is one of the most popular stochastic volatility models in the equity market, where the equity price $\left(S_{t}\right)_{0 \leq t \leq T}$ is modeled by

$$
\begin{cases}d S_{t}=r S_{t} d t+\sqrt{v_{t}} S_{t}\left(\sqrt{1-\rho^{2}} d W_{t}^{(1)}+\rho d W_{t}^{(2)}\right), & S_{0}>0 \\ d v_{t}=\kappa\left(\theta-v_{t}\right) d t+\sigma \sqrt{v_{t}} d W_{t}^{(2)}, & v_{0}=\sigma_{0}^{2}>0\end{cases}
$$


where $W^{(1)}$ and $W^{(2)}$ are independent Brownian motions defined on $\left(\Omega, \mathcal{F},\left(\mathcal{F}_{t}\right)_{t \in[0, T]}, P\right)$. Although the Heston model does not admit the analytical density function or distribution function, the log-asset price has the following characteristic function [13]

$$
\phi(\omega, t):=E\left[\exp \left(i \omega \log \left(S_{t}\right)\right) \mid S_{0}, v_{0}\right]=\exp (A+B+C),
$$

where

$$
\begin{array}{ll}
A=i \omega\left(\log \left(S_{0}\right)+(r-q) t\right), & d=\sqrt{(\rho \sigma \omega i-\kappa)^{2}+\sigma^{2}\left(\omega i+\omega^{2}\right)}, \\
B=\theta \kappa \sigma^{-2}\left((\kappa-\rho \sigma \omega i+d) t-2 \log \left(\left(1-g e^{d t}\right) /(1-g)\right)\right), & g=(\kappa-\rho \sigma \omega i+d) /(\kappa-\rho \sigma \omega i-d), \\
C=v_{0} \sigma^{-2}(\kappa-\rho \sigma \omega i+d)\left(1-e^{d t}\right) /\left(1-g e^{d t}\right) . &
\end{array}
$$

Given the model parameters

$$
\kappa=1.15, \quad \theta=0.0348, \quad \sigma=0.39, \quad r=0.04, \quad T=1, \quad v_{0}=0.0348, \quad S_{0}=10,
$$

we first recover the density function $f_{\log S_{1}}$ and the distribution function $F_{\log S_{1}}$ from the characteristic function (4.2) with the COS method. The truncation interval is set to be $[-10,5]$, and $2^{10}$ terms are used in the approximation formula for the density function. $f_{\log S_{1}}$ and $F_{\log S_{1}}$ work as the original density function and the distribution function, respectively. Sequentially, due to

$$
F_{S_{1}}(x)=F_{\log S_{1}}(\log x) \quad \text { and } \quad f_{S_{1}}(x)=\frac{1}{x} f_{\log S_{1}}(\log x)
$$

we can calculate the density function $f_{S_{1}}$ and the distribution function $F_{S_{1}}$ of $S_{1}$.

The truncation approximation (3.3) is set up for the distorted density function induced by the distortion functions listed in Table 1 with $\lambda=1$, respectively. The truncation interval for $S_{1}$ is set to be [0.01,40], and the number $(K)$ of the terms in (3.3) varies among $\left\{2^{5}, 2^{6}, 2^{8}\right\}$. The computation time and the values of the distorted expectations are reported in Table 2. On the other hand, ten million paths of the Heston model are simulated with the balanced Milstein scheme [14] at the step-size 0.01. The simulation costs about 37 seconds. The ordered sample of the terminal value can be saved and reused to calculate distorted expectations under different distortion functions. However, it still costs about 0.1 seconds to calculate the distorted expectations with the saved paths, while the T-COS method costs less than 0.1 milliseconds. It means that the T-COS method is still far more efficient than the MC method with saved paths, when the distortion function or its parameters vary. When the number $(K)$ of terms in the truncation approximation (3.3) increases, the increase in computation time is negligible ${ }^{1}$. Since the values of the distorted expectations corresponding to $K=2^{6}$ and $K=2^{8}$ are the same up to the current accuracy, we recommend to set $K=2^{6}$ in this setting. In other applications of the T-COS method, one may do the same test to choose an appropriate value for $K$. Since the T-COS method and the MC method provide quite similar values for the distorted expectation, the T-COS method is recommended for its extraordinary efficiency. The implementations are done in MATLAB (2014b) (Processor: Intel Core(TM) i7-3770 CPU @ 3.4GHz, RAM: 8GB).

Table 2: Distorted expectations $\mathbb{E}_{\Psi^{1}}\left[S_{1}\right](\mathrm{DE})$

\begin{tabular}{|c|l|l|l|l|l|l|l|l|}
\hline \multirow{2}{*}{} & \multicolumn{3}{|c|}{ MAXMIN } & \multicolumn{4}{c|}{ MINMAX } \\
\cline { 3 - 4 } & \multirow{2}{*}{$\mathrm{MC}$} & \multicolumn{3}{|c|}{ TCOS } & \multicolumn{3}{c|}{ T-COS } \\
\cline { 3 - 4 } \cline { 6 - 8 } & & $K=2^{5}$ & $K=2^{6}$ & $K=2^{8}$ & & $K=2^{5}$ & $K=2^{6}$ & $K=2^{8}$ \\
\hline time & $37 \mathrm{~s}$ & $0.04 \mathrm{~ms}$ & $0.05 \mathrm{~ms}$ & $0.07 \mathrm{~ms}$ & $0.1 \mathrm{~s}^{\dagger}$ & $0.04 \mathrm{~ms}$ & $0.04 \mathrm{~ms}$ & $0.07 \mathrm{~ms}$ \\
\hline DE & 8.105682 & 8.104594 & 8.105569 & 8.105569 & 7.588375 & 7.587122 & 7.588247 & 7.588247 \\
\hline
\end{tabular}

${ }^{\dagger}$ Calculated with the saved sample in the increasing order

To show the effect of the distortion function, we plot the distorted expectations under the distortion functions listed in Table 1 with different values of $\lambda$. As shown in Figure 1, the distorted expectation decreases with the distortion parameter $\lambda$. The higher the value of $\lambda$ is, the higher the stress level is. Note that different distortion functions lead to different approximations for the distorted expectation, especially at high stress levels.

${ }^{1}$ When the truncation interval for $S_{1}$ is enlarged to be $[0.01,50]$, the changes in computation time and the distorted expectations are also negligible. We do not report them here. These results will be provided on request. 


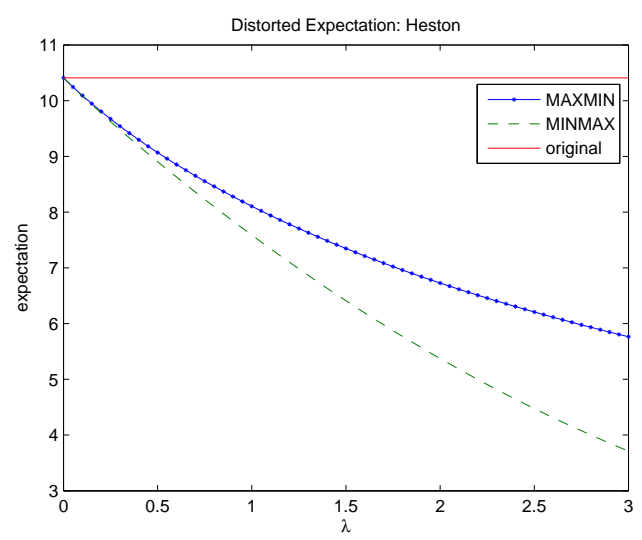

Figure 1: Distorted expectations $\mathbb{E}_{\Psi^{\lambda}}\left[S_{1}\right]$ associated with MINMAX and MAXMIN distortion functions at different stress levels $\lambda$.

\section{Conclusion}

In this paper, we proposed an analytical approximation, as an alternative to the Monte Carlo method, for the distorted expectations in the setting where the density function or the characteristic function of the risk factor is given in its analytical form.

The proposed method is based on a truncated sum of the Fourier-cosine series expansion for the distorted density function on a finite interval. The coefficients of the terms in the truncation approximation can be calculated with Algorithm 3.1 based on the FFT algorithm. Then, the resulting approximation for the distorted density function provides an analytical approximation for the distorted expectation. Numerical examples highlight that the proposed method is highly efficient and accurate.

Distorted expectations are associated with distortion risk measures, which play an important role in risk management. The proposed method can be used to quantify the risk of portfolios, as well as a single risk factor, with the risk measures beyond V@R and Expected Shortfall [18]. They also arise in portfolio optimization, conic finance and so on. The problems involving distorted expectations in these areas can be solved with our method under realistic model assumptions. Our ongoing research focuses on the application of the truncation approximation in the dynamic portfolio optimization under distortion risk measures.

Acknowledgements. The authors would like to thank an anonymous referee for the valuable suggestions and comments to improve the manuscript. Xianming Sun acknowledges the financial support by the China Scholarship Council under the CSC-grant (No. 201306370129) and by the Ghent University under the BOFcofunding for Chinese PhD students holding a CSC-grant. Xianming Sun also acknowledges the support by NSF of China (No. 11171352) and the Hunan Provincial Innovation Foundation for Postgraduate Students (No. CX2013B040).

\section{References}

[1] Albrecher, H., Guillaume, F., Schoutens, W., 2013. Implied liquidity: model sensitivity. Journal of Empirical Finance 23, $48-67$.

[2] Artzner, P., Delbaen, F., Eber, J., Heath, D., 1999. Coherent measures of risk. Mathematical Finance 9 (3), $203-228$.

[3] Barberis, N., Huang, M., 2008. Stocks as lotteries: the implications of probability weighting for security prices. American Economic Review 98 (5), 2066-2100.

[4] Boyd, J. P., 2000. Chebyshev and Fourier spectral methods, 2nd Edition. Dover Publications, Inc., New York.

[5] Carr, P., Madan, D., 1999. Option valuation using the fast Fourier transform. Journal of Computational Finance 2 (1990), $1-18$.

[6] Cherny, A., 2006. Weighted V@R and its properties. Finance and Stochastics 10 (3), 367-393.

[7] Cherny, A., Madan, D., 2009. New measures for performance evaluation. Review of Financial Studies 22 (7), $2571-2606$.

[8] Dhaene, J., Kukush, A., Linders, D., Tang, Q., 2012. Remarks on quantiles and distortion risk measures. European Actuarial Journal 2 (2), 319-328.

[9] Fang, F., Oosterlee, C., 2008. A novel pricing method for European options based on Fourier-cosine series expansions. SIAM Journal on Scientific Computing 31 (2), 826-848.

[10] Follmer, H., Schied, A., 2011. Stochastic finance, 3rd Edition. De Gruyter, Berlin, Newyork.

[11] Gasquet, C., Witomski, P., 1999. Fouier analysis and applications. Springer-Verlag, New York. 
[12] He, X. D., Zhou, X. Y., 2011. Portfolio choice under cumulative prospect theory: an analytical treatment. Management Science 57 (2), 315-331.

[13] Heston, S. L., 1993. A closed-form solution for options with stochastic volatility with applications to bond and currency options. Review of Financial Studies 6 (2), 327-343.

[14] Kahl, C., Schurz, H., 2006. Balanced Milstein methods for ordinary SDEs. Monte Carlo Methods and Applications 12 (2), 143-170.

[15] Lv, W., Pan, X., Hu, T., 2013. Asymptotics of the risk concentration based on the tail distortion risk measure. Statistics \& Probability Letters $83(12), 2703-2710$.

[16] Madan, D., Cherny, A., 2010. Markets as a counterparty: an introduction to conic finance. International Journal of Theoretical and Applied Finance 13 (08), 1149-1177.

[17] Madan, D. B., Pistorius, M. R., Stadje, M., 2015. On dynamic spectral risk measures and a limit theorem. working paper, $1-53$.

[18] Ortiz-Gracia, L., Oosterlee, C. W., 2014. Efficient VaR and expected shortfall computations for nonlinear portfolios within the delta-gamma approach. Applied Mathematics and Computation 244, 16-31.

[19] Sun, X., Vanmaele, M., 2015. Model-based and model-free upper bounds for discrete arithmetic Asian options. working paper, $1-27$.

[20] Tsukahara, H., 2013. Estimation of distortion risk measures. Journal of Financial Econometrics 12 (1), $213-235$.

[21] Wang, R., Ziegel, J. F., 2015. Elicitable distortion risk measures: a concise proof. Statistics \& Probability Letters $100,172-175$.

[22] Wang, S., 2002. A universal framework for pricing financial and insurance Risks. ASTIN Bulletin 32 (2), $213-234$.

[23] Yin, C., 2015. New class of distortion risk measure and their tail asymptotics with emphasis on VaR. arXiv:1503.08586, 1-35. 